\title{
Capturing Transient Electronic and Molecular Structures in Liquids by Picosecond X-Ray Absorption Spectroscopy
}

\author{
W. Gawelda ${ }^{1,2}$, V. T. Pham ${ }^{1}$, A. El Nahhas ${ }^{1}$, M. Kaiser ${ }^{1}$, Y. Zaushitsyn ${ }^{1}$, \\ S. L. Johnson ${ }^{2}$, D. Grolimund ${ }^{2}$, R. Abela ${ }^{2}$, A. Hauser ${ }^{3}$, C. Bressler ${ }^{1}$, M. Chergui ${ }^{1}$ \\ ${ }^{1}$ Laboratoire de Spectroscopie Ultrarapide (LSU), Ecole Polytechnique Fédérale de Lausanne (EPFL), BSP, \\ CH-1015 Lausanne, Switzerland \\ ${ }^{2}$ Swiss Light Source, Paul Scherrer Institut, CH-5232 Villigen-PSI, Switzerland \\ ${ }^{3}$ Département de Chimie Physique, Université de Genève, CH-1121 Genève, Switzerland
}

\begin{abstract}
We describe an advanced setup for time-resolved x-ray absorption fine structure (XAFS) spectroscopy with picosecond temporal resolution. It combines an intense femtosecond laser source synchronized to the x-ray pulses delivered into the microXAS beamline of the Swiss Light Source (SLS). The setup is applied to measure the short-lived high-spin geometric structure of photoexcited aqueous $\mathrm{Fe}(\mathrm{bpy})_{3}$ at room temperature.
\end{abstract}

Keywords: XAFS, XANES, EXAFS, picosecond time resolution, spin-crossover, transition metal compounds. PACS: $61.10 . \mathrm{Ht}$, 61.20.Lc, 72.80.Ga, 75.30.Wx, 82.53.-k, 78.70.Dm, 82.20.-w, 82.20.Kh, 82.50.-m

\section{INTRODUCTION}

Time-resolved X-ray absorption spectroscopy is a young field aiming to deliver transient chemical structures in the course of a chemical reaction [1]. This is accomplished via analyzing the x-ray absorption near edge structure (XANES), and the extended x-ray absorption fine structure (EXAFS) at a given time after photoexcitation. We have implemented our experimental scheme [2] at the microXAS beamline of the Swiss Light Source (SLS, Villigen) with upgrades, which we describe below. Finally, with this improved setup we have recorded the excited state XAFS of a short-lived spin-crossover compound, and measured its altered geometric structure.

\section{EXPERIMENTAL SETUP}

The setup at the microXAS beamline of SLS is shown in Fig. 1. X-rays from a $\mathrm{Si}(111)$ monochromator enter the experimental hutch, where they are focused to ca. $50 \mu \mathrm{m}$ diameter onto the sample with a pair of Kirk-Patrick Baez (KB) mirrors. $\mathrm{X}$-ray signals are detected with 4 large-area avalanche photodiodes (APD), one each for transmission $\left(\mathrm{I}_{1}\right)$ and for the incident signal $\left(\mathrm{I}_{0}\right)$ scattered off a thin $\mathrm{Cr}$ foil, and two fluorescence APDs $\left(\mathrm{I}_{\mathrm{F} 1}, \mathrm{I}_{\mathrm{F} 2}\right)$ for $\mathrm{x}$-ray fluorescence from the sample. The sample consists of a free-flowing liquid jet, which is excited with $400 \mathrm{~nm}$ light from an amplified fs laser system. Spatial overlap including a measurement of the spot sizes on the sample between both laser and $\mathrm{x}$-ray beams is set via steering the laser beam onto the $\mathrm{x}$-ray spot on sample and monitored with an imaging CCD camera.

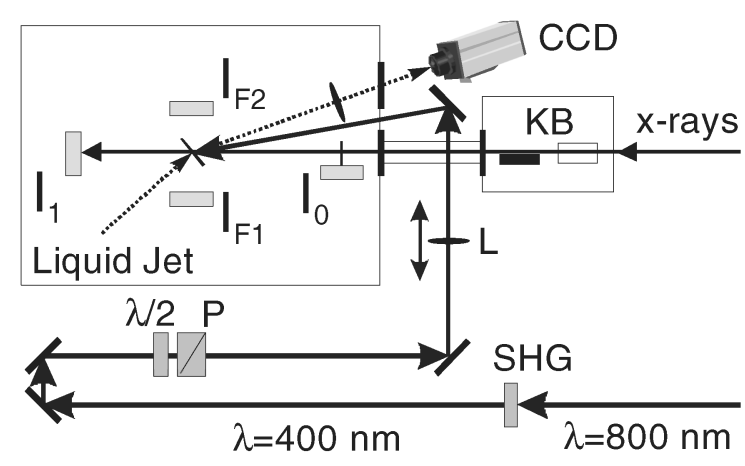

FIGURE 1. Experimental endstation with focused and overlapping laser and x-ray beams on the liquid sample. 4 detectors $\left(\mathrm{I}_{0}, \mathrm{I}_{1}, \mathrm{I}_{\mathrm{F} 1}, \mathrm{I}_{\mathrm{F} 2}\right)$ are used for measuring the XAFS, and an imaging CCD detector monitors spatial overlap and beam sizes. $800 \mathrm{~nm}$ light is frequency-doubled (SHG) and its pulse energy adjusted with a $\lambda / 2$ waveplate and polarizer $(\mathrm{P})$ combination before being focused (with the lens $\mathrm{L}$ ) onto the sample. The $\mathrm{x}$-ray beam is focused to ca. $50 \mu \mathrm{m}$ diameter with the KB optics.

CP882, X-ray Absorption Fine Structure-XAFS13 edited by B. Hedman and P. Pianetta

(C) 2007 American Institute of Physics 978-0-7354-0384-0/07/\$23.00 


\section{Data Acquisition Scheme}

We exploit a special electron bunch filling pattern at SLS for recording laser-pump-x-ray probe spectra (Fig. 2). The storage ring consists of 480 buckets, each separated by $2 \mathrm{~ns}$, in which 390 are filled (a filled bucket is also called an electron bunch) with ca. $6 \mathrm{x}$ $10^{9}$ electrons (thus generating each an average current of ca. $0.9 \mathrm{~mA}$ ), while in the subsequent $180 \mathrm{~ns}$ dark section a single densely packed (with up to 5 times more charge) hybrid electron bunch is placed. The $\mathrm{x}$ ray pulse from this electron bunch is used to probe the photoinduced changes of the $\mathrm{x}$-ray absorption inside the sample.

The amplified laser system for photoexcitation delivers $0.4 \mathrm{~mJ}, 400 \mathrm{~nm}, 120 \mathrm{fs}$ laser pulses at $1 \mathrm{kHz}$ repetition rate, which is synchronized to the round trip frequency ( $T=960 \mathrm{~ns}$ ), thus ensuring a constant time delay between the laser and selected probing $\mathrm{x}$-ray pulses. A gated integrator delivers a sequence of output signals proportional to the input signal integrated over a fixed time window (Fig. 3). These signals are recorded with an ADC card, triggered at 4 $\mathrm{kHz}$, in order to provide a sequence of the $\mathrm{x}$-ray signals when the laser is on and off, but also for recording the electronic background signal with no $\mathrm{x}$ rays present. First, the $\mathrm{x}$-ray signal at time $\Delta t$ after laser excitation is measured $\left(I_{p}\right)$, then a background signal without $\mathrm{x}$-rays is recorded after ca. $250 \mu \mathrm{s}$ ( $\mathrm{I}_{\text {bck }}(250 \mu \mathrm{s})$ ), which enables post-correcting baseline drifts in the gated integrator, and finally, after $0.5 \mathrm{~ms}$, the x-ray signal from a fresh sample without laser excitation is recorded $\left(\mathrm{I}_{\mathrm{u}}\right)$ followed by a baseline correction measurement $\left(\mathrm{I}_{\mathrm{bck}}(500 \mu \mathrm{s}+250 \mu \mathrm{s})\right.$, not shown in the figure). The pump-probe signal in transmission is defined as the transient absorption $T_{\text {tr }}$ of the photoexcited sample via

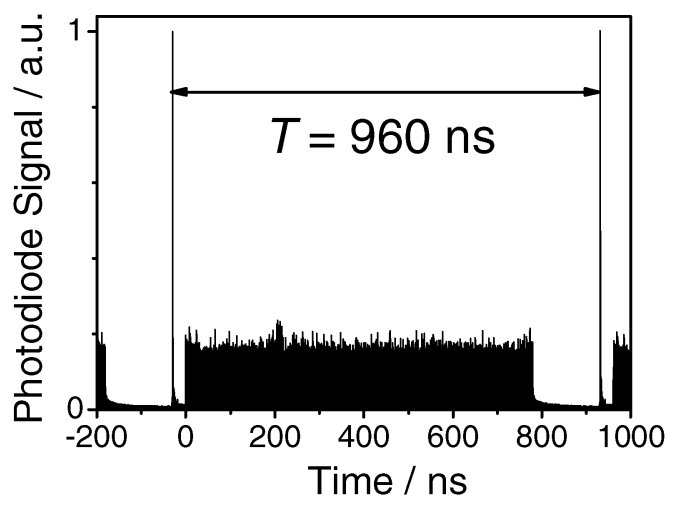

FIGURE 2. Bunch filling pattern at SLS. Trains of 390 electron bunches with $2 \mathrm{~ns}$ spacing are followed by a $180 \mathrm{~ns}$ long empty section, in which a densely packed electron bunch is placed $30 \mathrm{~ns}$ before the start of the bunch train.

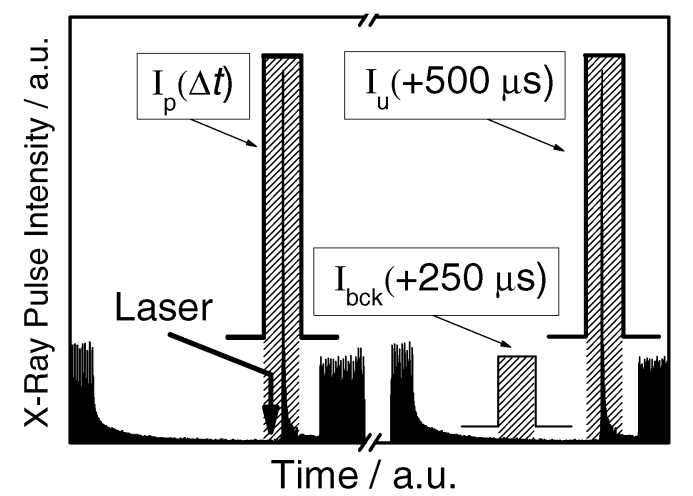

FIGURE 3. Data acquisition scheme employed at the microXAS beamline of the Swiss Light Source. A gated integrator measures sequentially the $\mathrm{X}$-ray intensity at the chosen time delay $\Delta t$, the background signal ca. $250 \mu$ s later, the x-ray signal of the unexcited sample after $500 \mu$ s (each within the indicated shaded time windows), and a second background signal without x-rays (not shown, $250 \mu$ s after $I_{u}$ ). This acquisition scheme is repeated for several thousand times per data point.

$$
T_{t r}(E, \Delta t)=\ln \left(\frac{I_{p u m}}{I_{u n p}}\right)
$$

with $I_{\mathrm{pum}}=I_{\mathrm{p}}(\Delta t)-I_{\mathrm{bck}}(\Delta t+250 \mu \mathrm{s})$ and $I_{\mathrm{unp}}=$ $I_{\mathrm{u}}(500 \mu \mathrm{s})-I_{\mathrm{bck}}(500 \mu \mathrm{s}+250 \mu \mathrm{s})$ being both the baseline $\left(I_{\text {bck }}\right)$ corrected photoexcited and unexcited $x$ ray signals, respectively (see Fig. 3).

The $\mathrm{x}$-ray signals are measured simultaneously in transmission and fluorescence yield modes (Fig. 1), using the same data acquisition strategy for all detectors, as shown in Fig. 3. The transient signal in fluorescence mode $T_{\mathrm{fl}}$ is defined via

$$
T_{f l}(E, \Delta t)=\frac{I_{p u m}^{f l}-I_{u m p}^{f l}}{I_{0}}
$$

with $I_{\mathrm{pum}}{ }^{\mathrm{fl}}, I_{\mathrm{unp}}{ }^{\mathrm{fl}}$ and $I_{0}$ being each baseline corrected as described above for transmission mode. Likewise, normalization of the unpumped spectra to the incident flux yields the static XAFS of the sample.

This data acquisition scheme permits us to measure the corresponding signals for every single incident $\mathrm{x}$ ray pulse, and for each data point (e.g., during a XAFS energy scan) a few thousand single $x$-ray pulse intensities are analyzed in order to store the averaged value and its standard deviation into the computer.

This treatment of the data permits us to assess the sensitivity of the experimental setup. Fig. 4 shows the pulse height distribution of $10^{3}$ single $\mathrm{x}$-ray pulses as recorded with the transmission APD and processed in the gated integrator. The measured width without beam (left) amounts to less than $20 \%$ of the width 
measured with $\mathrm{x}$-rays on the APD (at this x-ray intensity). This pulse height distribution can be analyzed according to [3]

$$
N=\left(\frac{N}{\Delta N}\right)^{2}=\left(\frac{N}{\sqrt{N}}\right)^{2} \geq\left(\frac{\bar{S}}{\sigma_{S}}\right)=N_{e f f}
$$

with $N$ being the true number of incident photons per pulse, and $\bar{S}$ the measured mean output value and its standard deviation (both in Volts). $N_{\text {eff }}$ then describes a lower limit for the incident number of photons per pulse, which becomes identical to $N$ only in the complete absence of additional (e.g., electronic) noise sources.

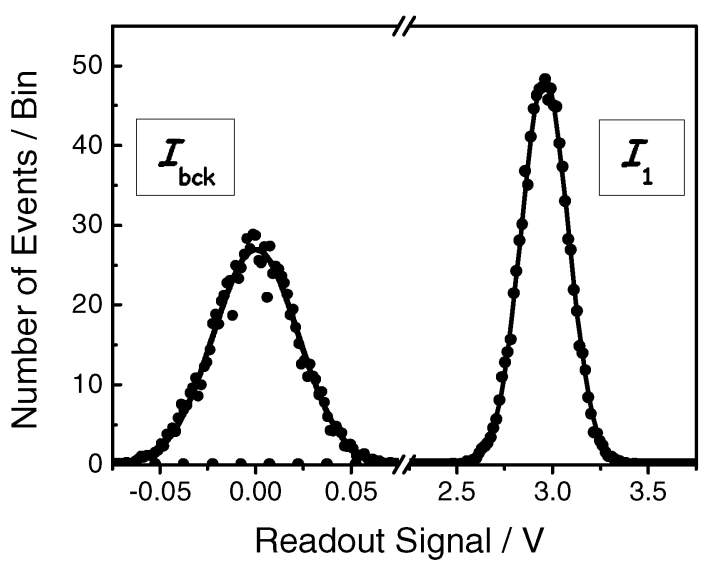

FIGURE 4. Pulse height distribution of $10^{3}$ recorded single $\mathrm{X}$-ray pulses without $\mathrm{X}$-radiation $\left(=I_{\mathrm{bck}}\right.$, left $)$ and with ca. 600 photons per pulse at $7 \mathrm{keV}\left(=I_{1}\right.$, right $)$ together with gaussian fits. The binning was ten-fold denser for the background distribution.

We have compared the value shown in Fig. 4 with the beamline specifications, and after correcting for various additional factors, e.g., sample and air transmission, we find that both values are identical to within less than a factor of two.

For fluorescence yield detection we enter the single photon counting regime, as seen in Fig. 5, showing the pulse height distributions for ca. 0.6 and 3 photons per pulse. In this case the background signal has the same width as a single photon event. Nevertheless, we can reliably distinguish between one- and more photon events, and by fitting each event with a Gaussianshaped function we can calculate the probability for each event. With this we can analyze the data according to the expected Poisson distribution, which is nicely confirmed (see insets in Fig. 5). As before, the average (and standard distribution) of such a distribution is recorded for each data point during a spectral scan.

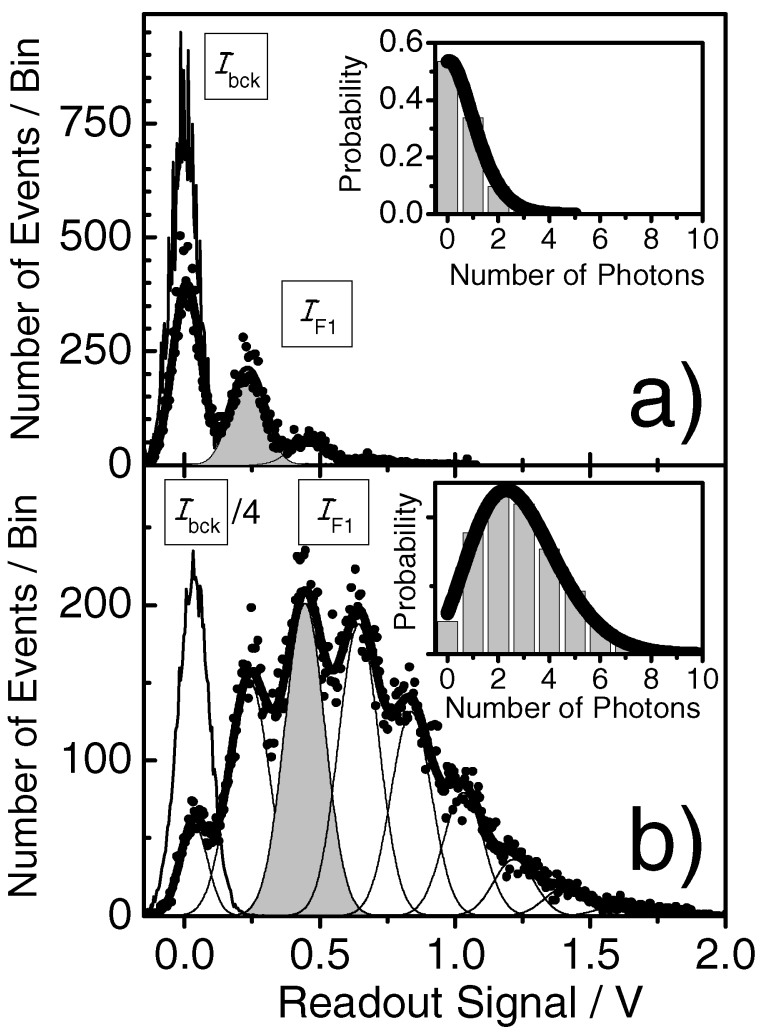

FIGURE 5. Pulse height distribution of ca. $10^{4}$ recorded single $\mathrm{x}$-pulses with the fluorescence detectors for two selected intensities, 0.624 (a) and 2.82 (b) photons per pulse. The background signal without $\mathrm{x}$-rays is shown as well. The insets show the analysis for a Poisson distribution including its fit curve.

It is interesting to note that this acquisition scheme exploiting gated APDs permits us to measure actual intensities for a wide range of pulse intensities. While Fig. 5 shows that we can reliably detect less than one photon per pulse, we have previously confirmed the linearity of APDs up to $6 \times 10^{4}$ photons/pulse [1]. Especially in fluorescence XAFS one usually operates the detectors with single photon counters preceded by a (constant fraction) discriminator. This procedure ignores two- and more photon events, and can only be approximately corrected in a postcalibration measurement. The current scheme, on the other hand, always delivers accurate values and therefore we can move the detectors rather close to the sample thus increasing its solid angle, but due to the limited energy resolution of the detector we can not distinguish between scattered and fluorescent light. The background signal due to scattered light was reduced to $<10 \%$ of the edge jump signal by placing the detectors within the direction of the x-ray polarization.

Finally, we have also confirmed the spatial overlap by scanning the laser beam across the overlap region during an actual pump-probe measurement (Fig. 6). 
Hereby the pump laser beam was scanned laterally across the overlap region by the last mirror before the sample (Fig. 1). The signal shown in the figure is the transient absorption measured in fluorescence mode. This confirms the actual pump-probe profile as predetermined with the imaging $\mathrm{CCD}$ by measuring the laser and x-ray spot sizes individually.

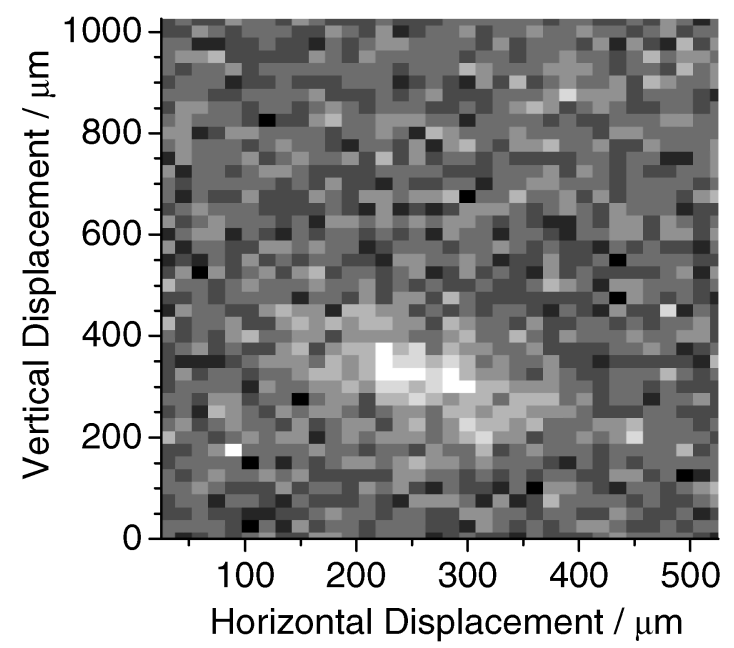

FIGURE 6. Spatial pump-probe profile during an actual time-resolved XAFS measurement $(\Delta t=50 \mathrm{ps})$. This image has been created by monitoring the transient absorption signal in fluorescence mode, while scanning the pump laser beam across the overlap region.

\section{TIME-RESOLVED XAFS STUDIES}

Iron(II)-tris-bipyridine, $\left[\mathrm{Fe}^{\mathrm{II}}(\mathrm{bpy})_{3}\right]^{2+}$ represents a typical example of a low spin (LS) compound with the characteristic energy level scheme shown in Fig. 7. In compounds with a sufficiently low adiabatic energy difference, $\Delta \mathrm{E}_{\mathrm{HL}}^{0}$, between $\mathrm{LS}$ and high spin (HS) states, that is, on the order of $100-1000 \mathrm{~cm}^{-1}$, spin crossover ( $\mathrm{SCO})$ can be temperature-induced. In the case of $\left[\mathrm{Fe}^{\mathrm{II}}(\mathrm{bpy})_{3}\right]^{2+}, \Delta \mathrm{E}_{\mathrm{HL}}^{0} \approx 6000 \mathrm{~cm}^{-1}$ and $\mathrm{SCO}$ can only be optically triggered and stabilized to some extent at cryogenic temperatures, in the so-called Light-Induced Excited Spin State Trapping (LIESST) process [4,5]. However, in contrast to SCO compounds with low-temperature lifetimes of the light-induced state of more than $10 \mathrm{~h}$, for the LS system typical values are on the microsecond timescale. The absorption spectrum of $\left[\mathrm{Fe}^{\mathrm{II}}(\mathrm{bpy})_{3}\right]^{2+}$ is characterized by an intense broad band centred at 520 $\mathrm{nm}$ due to the singlet Metal-to-Ligand-ChargeTransfer $\left({ }^{1}\right.$ MLCT) state. Photoexcitation into this band is followed by a cascade of intersystem crossing steps through singlet, triplet and quintet MLCT and ligandfield (LF) states, which brings the system to the lowest-lying (HS) quintet state, ${ }^{5} \mathrm{~T}_{2}$, with almost unit quantum yield in $<1$ ps (Fig. 1) [5]. This state relaxes non-radiatively to the LS ground state within $\sim 0.6 \mathrm{~ns}$ in aqueous solutions at room temperature.

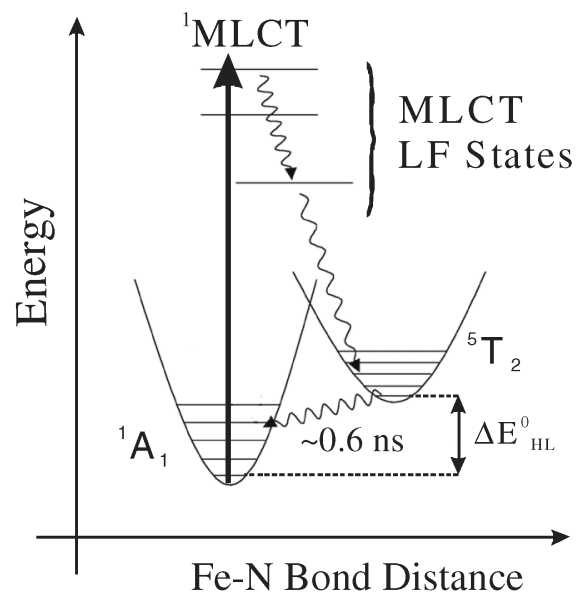

FIGURE 7. Energy-level scheme of $\mathrm{Fe}(\mathrm{bpy})_{3}$ with its photochemical cycle. A laser pulse excites the system into its metal-to-ligand charge transfer (MLCT) state, from which it cascades over MLCT and metal-centered ligand field (LF) states into the high spin (HS) ${ }^{5} \mathrm{~T}_{2}$ state. In room temperature aqueous solutions it relaxes nonradiatively back to the ground state within $0.6 \mathrm{~ns}$.

Fig. 8a shows the static XAS spectrum of the first $200 \mathrm{eV}$ above the $\mathrm{Fe} \mathrm{K}$ absorption edge of a $25 \mathrm{mM}$ aqueous solution of $\left[\mathrm{Fe}^{\mathrm{II}}(\mathrm{bpy})_{3}\right]^{2+}$. It is characterized by a number of XANES features (labeled A to D), that have already been discussed for similar Fe-compounds [6]. The features that lie $50 \mathrm{eV}$ above the edge are all electron scattering features, in particular the E-feature, which is dominated by scattering from the nearby $\mathrm{N}$ atoms. The exact assignment of the XANES features will not be discussed here, but all have been shown to undergo significant changes upon SCO [6-9]. This is also the case here, as can be seen in fig. $8 b$, which shows the transient difference spectrum measured 50 ps after excitation. In particular, the $\mathrm{B} / \mathrm{C}$ features gain intensity, while shifting to lower energy. The D-band intensity decreases, while the E-feature shifts to lower energies. Most of these changes are attributed to the metal-ligand bond changes. Additional changes in the high energy region (not shown here) are also clearly observed, which clearly point to a significant $\mathrm{Fe}-\mathrm{N}$ bond change. That these changes are due to the photoinduced HS complex is seen in Fig. 9, which compares the time evolution of the absorption changes at the B feature with the kinetics of ground state recovery measured by optical pump-probe spectroscopy. The latter reflects the repopulation of the LS state by the decay of the HS state and it perfectly matches the $\mathrm{x}$-ray data. 


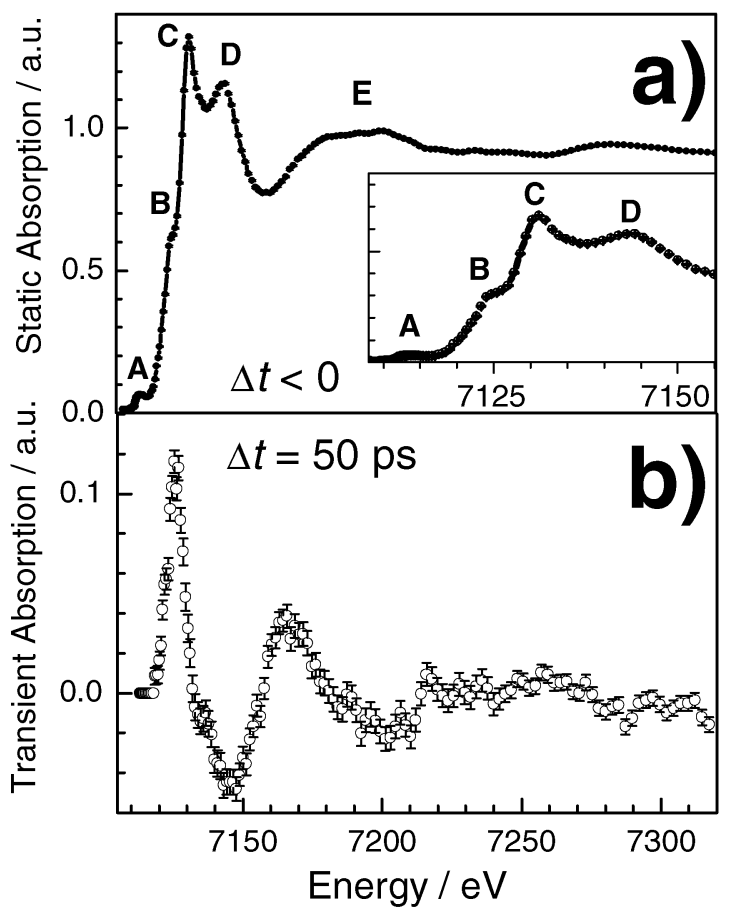

FIGURE 8. a) LS ground state XAFS spectrum of $\left[\mathrm{Fe}(\mathrm{bpy})_{3}\right]^{2+}$ (the inset zooms into the edge region) b) Transient XAFS after 50 ps.

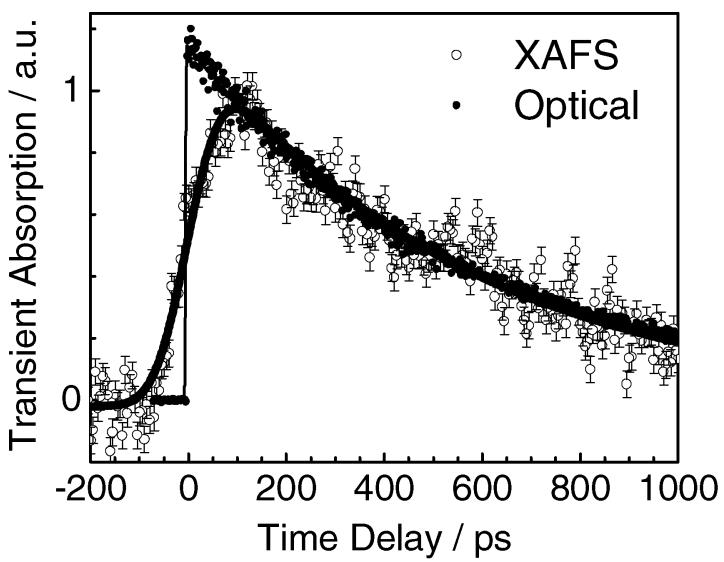

FIGURE 9. Kinetics of the difference XAS signal of aqueous $\mathrm{Fe}$ (bpy) 3 at room temperature, recorded at $7126 \mathrm{eV}$ (open circles) upon $400 \mathrm{~nm}$ excitation, and that of the optical signal (triangles) recorded in transmission at $523 \mathrm{~nm}$, and reflecting the repopulation of the ground state together with fit curves. The solid lines represent the fit curves, which in the case of the $\mathrm{X}$-ray signal is a monoexponential convoluted with the 100 ps width of the X-ray pulse.

The difference absorption spectrum (Fig. 8b) is defined as [1]

$T(\mathrm{E}, t)=f(t) \cdot\left[A_{\mathrm{HS}}(E, t)-A_{\mathrm{LS}}(E)\right]$
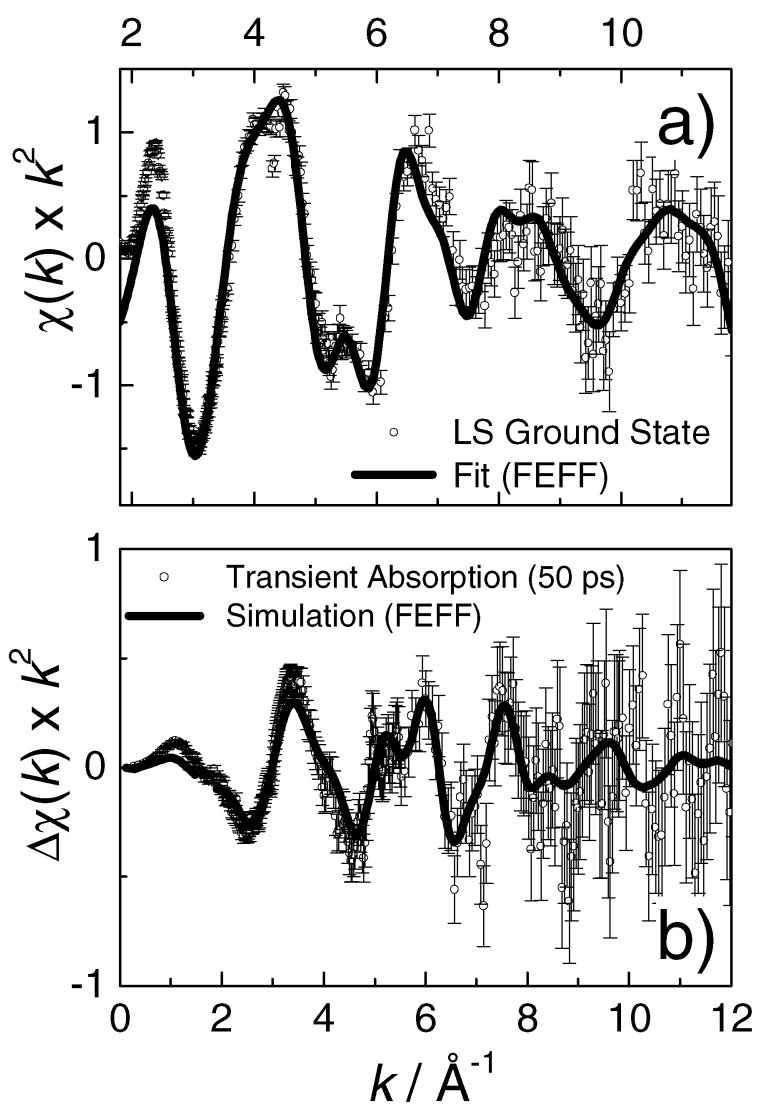

FIGURE 10. a) LS state EXAFS spectrum together with the fit curve yielding $R_{\mathrm{Fe}-\mathrm{N}}=1.97(0.01) \AA$. b) Transient EXAFS after 50 ps together with a transient simulation for $\Delta R_{\mathrm{Fe}-\mathrm{N}}=$ $0.18 \AA$.

where $f(\mathrm{t})$ is the fractional population of the HS complex at time $t(50 \mathrm{ps}$ in fig. $2 \mathrm{~b}), A_{\mathrm{LS}}(E)$ is the absorption spectrum of the LS complex (Fig. 8a), and $\mathrm{A}_{\mathrm{HS}}(\mathrm{E}, t)$ that of the HS complex, at the time $t$ following the photoexcitation. In order to extract the excited state structure correctly, $f(\mathrm{t})$ must be known, and we measured a value of $22(2) \%$ at $t=50 \mathrm{ps}$, in laser-only pump-probe experiments carried out under identical experimental conditions as in the present measurements.

We have used these values for a simulation of the transient EXAFS spectrum shown in Fig. 10. In Fig. 10a the static EXAFS has been compared with the fitted outcome of the EXAFS calculation performed with the FEFF 8.20 code over a large energy range (ca. $650 \mathrm{eV}$ above the edge). The results yield an excellent agreement with the LS structure as determined by static x-ray crystallography. The fit was done by taking into account all scattering contributions and by varying their Debye-Waller factors to best match the experimental data. In addition, we have refined the Fe$\mathrm{N}$ bond distance by setting it as a free running 
parameter and the obtained result agrees very well with the published value of $1.97 \AA$ within its uncertainty of $0.01 \AA$.

In the next step, we have performed several EXAFS calculations for several structural models with different Fe-N distances (the actual models were obtained by moving all 3 bpy ligands symmetrically outwards the Fe atom and thus modifying the bond lengths of all constituent atoms with respect to the absorber) in steps ranging from 0.03 to $0.005 \AA$. We then calculated the transient EXAFS using $f=22 \%$, and the obtained ground state fit result from Fig. 10a. An excellent agreement is obtained for $\Delta R_{\mathrm{Fe}-\mathrm{N}}=0.18$ $\AA$, and the resulting transient simulation is depicted in Fig. 10b together with the data. However, the uncertainty of this bond length increase has not been determined in this treatment, so the actual value may differ somewhat.

We are currently implementing a fit procedure to reliably quantify the actual bond length increase, as measured in the transient absorption spectra. This novel approach may yield uncertainties on the order of conventional state-of-the-art EXAFS studies, i.e., in the 0.01-0.001 $\AA$ range. This is because transient structural changes are rather small compared to the ground state structure. With the precise knowledge of the ground state structure (e.g., from crystallographic measurements), the fit procedure for determining the excited state becomes less ambiguous. Using the transient XAFS instead of the excited state XAFS spectrum can then deliver much more precise values for the structural changes.

In summary, we have reported on the structural and electronic changes of a short-lived intermediate of a photocycle via picosecond $\mathrm{x}$-ray absorption spectroscopy. Currently, a bond length increase of $0.18 \AA$ seems reasonable both from the comparison of the transient spectra as well as from theoretical calculations. A full multiple scattering analysis of the photoinduced changes in the XANES and of the EXAFS region is currently underway, which should deliver a precise value for the $\mathrm{Fe}-\mathrm{N}$ bond distance change.
The physical time scale for relaxation from the singlet ${ }^{1}$ MLCT to the HS state is expected to be $<1 \mathrm{ps,}$ occuring via several intermediate singlet and triplet states, which are each accompanied by a structural modification of the $\mathrm{Fe}-\mathrm{N}$ bond distances. Optical spectroscopy has so far not been able to resolve these steps, and is unable to determine their structures. Future extension of these experiments using femtosecond $\mathrm{x}$-ray pulses may soon provide better insight into this relaxation process.

\section{ACKNOWLEDGMENTS}

This work is funded by the Swiss National Science Foundation (FNRS), via contracts 620-066145, 200021-107956 and 200021-105239 and by the Swiss Light Source (SLS). We thank the microXAS beamline staff, M. Willimann, M. Harfouche and B. Meyer, for their assistance and help during the measurements.

\section{REFERENCES}

1. C. Bressler and M. Chergui, Chem. Rev. 104, 1781 (2004).

2. M. Saes, W. Gawelda, R. Abela, D. Grolimund, F. van Mourik, S. L. Johnson, P. A. Heimann, R. W. Falcone, R. W. Schoenlein, A. M. Lindenberg, M. Chergui, C. Bressler, Rev. Sci. Instrum. A 75 (1), 24-30 (2004).

3. W. Gawelda, C. Bressler, M. Saes, et al., Physica Scripta T115, 102 (2005).

4. A. Hauser, Topics in Current Chemistry 234, 155 (2004).

5. C. Brady, J. J. McGarvey, J. K. McCusker, et al., Topics in Current Chemistry 235, 1 (2004).

6. C. Hannay, M. J. HubinFranskin, F. Grandjean, et al., Inorganic Chemistry 36, 5580 (1997).

7. a) H. Oyanagi, T. Tayagaki, and K. Tanaka, Journal of Physics and Chemistry of Solids 65, 1485 (2004), b) H. Oyanagi, T. Tayagaki, and K. Tanaka, Journal of Luminescence 119, 361 (2006).

8. a) J. Kusz, H. Spiering, P. Gütlich, J. Appl Cryst. 33, 201 (2000), b) J. Kusz, D. Schollmexer, H. Spiering, P. Gütlich, J. Appl. Cryst. 38, 528 (2005).

9. M. L. Boillot, J. Zarembowitch, J. P. Itie, et al., New Journal of Chemistry 26, 313 (2002). 